\title{
Actualización de la cirugía oral en el paciente anticoagulado
}

\author{
J. Giner Díaz¹, A. González Corchón¹, V. Ordóñez Soblechero¹, \\ R. Martín-Granizo², A. Berguer ${ }^{3}$
}

\begin{abstract}
Resumen: Introducción: En el momento actual y sólo en el Reino Unido, alrededor de 300.000 personas están sometidas a anticoagulación oral. Con una población en claro envejecimiento en Europa y Norte América y una preocupación cada vez mayor en lo que especta a la salud dental, el número de pacientes anticoagulados de forma oral que requerirán algún tipo de cirugía oral está sujeto a incrementarse. Este estudio pretende proponer una serie de directrices a la hora de plantear el manejo de un paciente anticoagulado que va a requerir cualquier tipo de cirugía oral. Material y método: Se ha revisado y comparado la literatura más reciente indexada en Medline. Además se han consultado diferentes libros de texto sobre la materia.

Resultados: Tradicionalmente, los pacientes anticoagulados que van ser sometidos a cirugía oral, venían siendo sometidos a una interrupción de su terapia o a una disminución de los niveles de anticoagulación antes del procedimiento quirúrgico. Fenómenos trombóticos y oclusiones vasculares son problemas comunes derivados de esta actitud. Incluso se han descrito algunos casos de embolia fatal. En los últimos años, en muchos pacientes se ha venido sustituyendo la coaguloterapia oral por una terapia de heparina parenteral estándar. Sin embargo, hay un importante debate suscitado sobre la idoneidad e, incluso, la seguridad de esta medida. Recientes publicaciones muestran que extracciones de piezas dentarias, y otro tipo de procedimientos quirúrgicos orales, pueden ser realizados sin modificar para nada la anticoagulación, si el INR (international normalized ratio) del paciente es de 4 o menor y se utilizan procedimientos especiales de hemostasia local. No se objetivan diferencias en cuanto al sangrado postoperatorio.

Discusión: Aunque se requieren más estudios en esta materia, puede considerarse que el manejo del paciente anticoagulado sometido a cirugía oral debe estar influenciado por diversos factores como el tipo de procedimiento quirúrgico, el valor INR, otros factores de riesgo concomitantes y una importante dosis de juicio clínico.

Conclusiones: Pensamos que el INR es un excelente índice que permite al cirujano conocer el estado de anticoagulación del paciente. Se proponen unas directrices para el manejo quirúrgico de este tipo de pacientes. Ante cualquier sombra de duda, debe buscarse el consejo del hematólogo 0 el médico prescriptor de la terapia.
\end{abstract}

Palabras clave: Cirugía oral; Anticoagulación; Anticoagulación; Hemostasia; Heparina.

Abstract: Introduction: At this time, and only in the United Kingdom, about 300,000 people are taking oral anticoagulants. With an aging population in Europe and North America, and a greater worryness about teeth health, the number of oral anticoagulated patients requiring oral surgery is likely to increase. It is the aim of this study to give some guidelines in order to deal with the anticoagulated patient who requires oral surgery in its different ways.

Matherial and methouds: The pertinent literature more recently published and indexed in Medline has been reviewed and compared. Besides, different surgery and clinics textbooks about the matter have been consulted.

Results: Traditionally, anticoagulated patients requining oral surgery were managed by either stopping their treatments or decreasing their anticoagulant levels before the surgical procedure. Common problems seen with this attitude were thrombi and vascular occlusion. Even, some cases of fatal emboli have been described. In the last years, some patients are converted from oral coagutherapy to parenteral standard heparin therapy before surgery. However, there is an important debate currently on whether this is required or even if it is safe. Recent publications show how teeth removal and other oral surgery procedures can be performed without modifying the anticoagulation regimens with INR (international normalized ratio) of 4 or less if special procedures for local haemostasis are used. No discernible differences in the postoperative outcome with regard to haemorrhage are found.

Discussion: Even though further prospectives studies are needed in this area, it can be considered that the management of oral surgery on patients treated with anticoagulants should be influenced by different factors, such as type of surgical procedure, the INR value, other concomitant risk factors and a great deal of clinical judgement.

Conclusions: We accept INR as an excellent method that allows the surgeon to know about the state of a patient's anticoagulation. Different guideliness for these patients' management are suggested. Any doubt should be referred to the haematologist or the physician prescriber.

Key w ords: Oral surgery; Anticoagulation; Haemostasis; Heparin.

1 Médico Residente

2 Médico Adjunto

3 Jefe de Servicio

Servicio de Cirugía Oral y Maxilofacial

(Jefe de Servicio: Dr. A. Berguer). Hospital Clínico San Carlos,

Madrid. España.

\section{Correspondencia:}

Dr. Jorge Giner

Avda Donostiarra 14-10-4

28027 Madrid, España.

E-mail: docginer@terra.es

\section{Introducción}

De forma amplia, podemos definir hemostasia como <el mecanismo de defensa del organismo que tiene por objeto evitar la hemorragia (o detenerla si se ha producido), mantener la integridad de la pared vascular y restablecer la circulación sanguínea cuando se ha obstruido un vaso». Para ello, consta de cuatro bien diferenciadas, aunque interrelacionadas:

1.- Vasoconstricción local 


\section{2.- Formación del trombo plaquetario: Adhesión y agregación.}

3.- Formación del trombo de fibrina: Coagulación

4.- Disolución del coágulo: Fibrinólisis

La coagulación es, como vemos, una fase de la hemostasia que tiene como finalidad la transformación del fibrinógeno (proteína soluble que actúa como componente de unión entre plaquetas en el trombo primario) en fibrina, que es ya una proteína insoluble que formará mallas dotando de estabilidad y resistencia al agregado. Esta transformación tiene lugar a través de la activación secuencial en «cascada»de una serie de factores plasmáticos que habitualmente se encuentran inactivos en la circulación. Ello sucede a través de dos vías: la intrínseca, en la que todos los factores implicados se encuentran en el torrente circulatorio, y la extrínseca, que se activa por un factor tisular (Fig. 1). Si no tuviera lugar la coagulación, el trombo inicial, compuesto en su mayor parte de plaquetas y fibrinógeno, sería, literalmente, barrido de la circulación sanguínea en un muy corto espacio de tiempo. ${ }^{1}$

Un paciente puede presentar alteraciones en la coagulación de muy diversa índole, tanto por exceso (estados de hipercoagulabilidad), como por defecto. La causa más frecuente de alteraciones de la coagulación en países desarrollados es la toma de fármacos. Aunque múltiples drogas interfieren o pueden hacerlo, con la coagulación, en este estudio nos vamos a ocupar de los pacientes sometidos a anticoagulación oral (ACO) o parenteral como parte de tratamiento de diversas afecciones (fibrilación auricular, válvulas cardíacas protésicas, trombosis venosa profunda y tromboembolismo pulmonar, cardiopatía isquémica, etc.).

El número de personas sometidas a ACO en el Reino Unido se estima en tomo a las $300.000,{ }^{2}$ pudiendo calcularse el número de pacientes en España, a tenor de la proporcionalidad, en torno a los 250.000. Los anticoagulantes orales utilizados en la actualidad son derivados cumarínicos, de los que existen tres tipos: Warfarina (Aldocumar ${ }^{\circledR}$, poco utilizado en nuestro país, aunque es el más usado en países anglosajones), Acenocumarol (Sintrom ${ }^{\circledR}$ ) y Fenindiona (no comercializado en España). Todos ellos son muy hidrosolubles y se absorben con rapidez en estomago y tracto intestinal alto, alcanzando el pico de concentración en plasma entre los 60 y 90 minutos de la administración. El anillo cumarínico antagoniza el efecto de la vitamina $\mathrm{K}$ mediante su unión al enzima vit $\mathrm{K}$ 2,3epóxido reductasa en el microsoma hepático; esto, en consecuencia, interfiere con la síntesis de los factores de la coagulación vit $\mathrm{K}$ dependientes: II, VII, IX, $\mathrm{X}$, proteínas C y S. Por tanto, se van a ver alterados los tiempos de protrombina (TP), y de tromboplastina parcial activada (APTT). ${ }^{3}$

Tras la administración de cumarínicos, el efecto terapéutico comienza en torno a las 8-12 horas, es

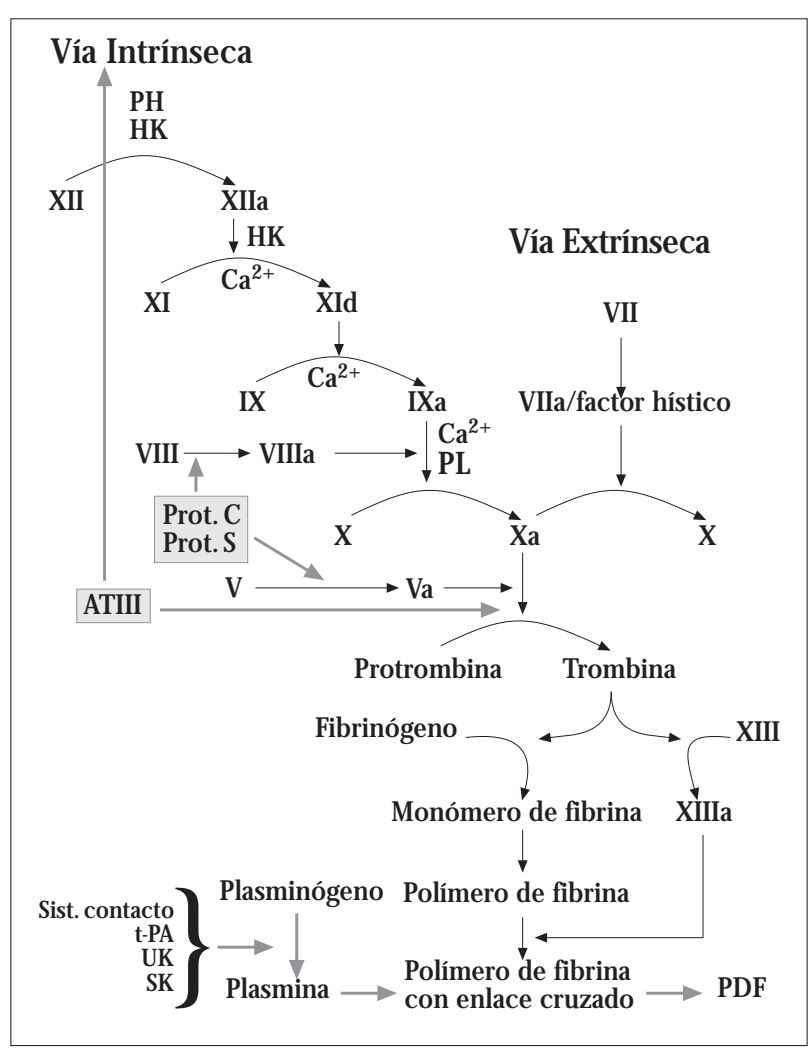

Figura 1. Cascada de la coagulación ${ }^{1}$

máximo a las 36 horas y persiste alrededor de 72 horas. ${ }^{4,5}$ Se metabolizan, fundamentalmente, en hígado, mediante el complejo citocromo p450 y su efecto es reversible con la vitamina K. La terapia ACO requiere una monitorización seriada; lo que se pretende es mantener el TP dentro de un rango terapéutico (entre 1,5 y 2,5 veces el valor control, en función de la patología). Desde 1983 se viene empleando el INR (International Normalized Ratio) que es la propoción entre el TP del paciente y un TP control, estandarizado y corregido para cada lote de reactivos utilizados, lo que permite comparar el resultado entre los distintos laboratorios. Normalmente el INR debe mantenerse entre 2 y 3 (a mayor INR, más anticoagulado está el individuo). En situaciones especiales (trombosis recidivantes, válvula cardíaca protésica o trombosis por anticoagulante lúpico circulante), se recomienda un mayor grado de anticoagulación (INR entre 3 y 4) (Tabla 1).1,3

La heparina es un agente anticoagulante parenteral que suele utilizarse para tratar episodios tromboembólicos agudos o como profilaxis de los mismos en pacientes hospitalizados. Se trata de un glicosaminoglicano sulfatado que actúa sobre la coagulación al fijarse a la antitrombina III (AT III), sobre la que actúa como importante cofactor inactivando así la trombina. Sobre las plaquetas, aunque es un débil proagregante, en presencia de AT III, inhibe fuertemente su activación y todas las funciones plaquetarias sub- 
Tabla 1. Indicaciones comunes para la terapia anticoagulante ${ }^{5}$

\begin{tabular}{|c|c|c|}
\hline Indicaciones & INR & $\begin{array}{c}\text { Duración } \\
\text { de la terapia }\end{array}$ \\
\hline Profilaxis de trombosis venosa & $2,0-3,0$ & Perioperatorio \\
\hline Tratamiento de trombosis venosa & & 3-6 meses \\
\hline Tratamiento de embolia pulmonar & & 3-6 meses \\
\hline Profilaxis de embolia sistémica & & Variable \\
\hline Válvula cardíaca & & \\
\hline Fibrilacion auricular & & \\
\hline Embolia sistémica recurrente & $\gamma$ & \\
\hline Post IAM & $2,5-3,5$ & \\
\hline $\begin{array}{l}\text { Embolia sistémica recurrente } \\
\text { en tto con heparina }\end{array}$ & & \\
\hline Prótesis valvulares & & De por vida \\
\hline Aórtica & $v$ & \\
\hline Mitral & $3,0-4,0$ & \\
\hline Aórtica + mitra & & \\
\hline
\end{tabular}

siguientes. Además, se han descrito varios casos de trombopenia autoinmune, por lo que en terapias de más de 5 días son necesarios recuentos plaquetarios. Tiene un efecto inmediato, pero dura tan sólo unas 46 horas, por lo que no suele ser preciso revertir su efecto (aunque, con este fin, puede utilizarse sulfato de protamina a dosis de $1 \mathrm{mg} / 100$ unidades). Su vida media se estima en torno a los 60 minutos, aunque es tremendamente variable en cada caso. En dosis altas puede producir una elevación de TP, INR y tiempo de hemorragia (TH); sin embargo, para la monitorización se utiliza el APTT (debe mantenerse en un rango definido, entre 1,5 y 2,5 veces el valor control).3,5,6

En los últimos 20 años, se vienen utilizando las heparinas de bajo peso molecular (HBPM) (dalteparina, ardeparina, enoxaparina, etc.) fundamentalmente para profilaxis de complicaciones tromboembólicas en cirugía vascular y pacientes hospitalizados; sin embargo, fue en 1998 cuando se aprobó su uso como tratamiento extrahospitalario de la enfermedad tro mboembólica pulmonar (TVP/EP). Las HBPM tienen diferentes propiedades que las heparinas no fraccionadas, lo que le confiere ciertas ventajas: fundamentalmente, se absorben de forma más completa por vía subcutánea (sc.), lo que, unido a su escasa fijación a las proteinas plasmáticas, tiene como consecuencia una mayor biodisponibilidad. También son menos potentes y tienen una vida media mayor. Todo esto hace que la dosis necesaria para lograr el efecto anticoagulante sea menor y que el paciente pueda ser manejado en su domicilio, con una inyección sc. diaria y sin necesidad de pruebas de coagulación. Además, apenas alteran el APTT y dan lugar, con menor frecuencia, a hemorragias postquirúrgicas.3,5,7 Diversos estudios (entre ellos un meta-análisis) no han encontrado diferencias significativas con las heparinas no fraccionadas en el tratamiento y prevención de la TVP/EP y sí una menor frecuencia de hemorragias importantes. ${ }^{7-9}$ Por tanto, las HBPM se han convertido en una formidable alternativa a la hospitalización. Entre sus desventajas están: la falta de costumbre en su uso por parte del paciente, lo que puede disminuir la adherencia terapéutica; la peor reversibilidad del efecto con la protamina; y, en cuanto a su uso alternativo a la ACO en cirugía oral en pacientes anticoagulados, la falta de estudios experimentales al respecto..$^{10}$

De todo lo anterior se deduce que cualquier paciente anticoagulado, sobre todo en terapia oral, que vaya a ser sometido a una intervención quirúrgica, puede convertirse en una verdadera prueba de fuego para su cirujano, anestesiólogo y médico prescriptor de la ACO. Esto, en al ámbito de la cirugía oral, tiene especial relevancia debido, principalmente, a la frecuencia con que la población se somete a este tipo de intervenciones. Además, la cirugía induce, de por sí, una serie de cambios en el entorno de la cavidad oral: inicialmente, la sangre y el exudado de la herida, debido a la presencia en ellos de una serie de inhibidores de la fibrinólisis, tienen una actividad procoagulante; pero al reducirse el sangrado, entra en juego la actividad fibrinolítica de la saliva por su contenido en plasminógeno y activador tisular del plasminógeno. ${ }^{11}$

Proponemos, en este estudio, unas directrices de actuación para la cirugía oral en el paciente anticoagulado basadas, fundamentalmente, en el estado de anticoagulación del paciente (INR), el tipo de cirugía, etc. No pueden recomendarse unas normas estrictas de actuación debido, principalmente, a la falta de consenso en la literatura al respecto así como a la idiosincrasia de cada caso, cada hospital o clínica, etc.

\section{Material y método}

Se han revisado numerosos artículos sobre la materia publicados en revistas internacionales durante los últimos años. Así mismo, se han consultado textos y publicaciones de reuniones de consensos de diversos organismos. Se ha realizado un análisis comparativo de la bibliografía seleccionada a fin de poder proponer una serie de directrices.

\section{Resultados}

Tradicionalmente cuando un paciente en tratamiento con ACOs va a ser sometido a cualquier cirugía oral, la actitud que se viene tomando consiste en interrumpir la terapia dos o tres días antes, determinar el INR el día de la intervención y realizar ésta sólo si se encuentra por debajo de un «umbral de seguridad»arbitrario (habitualmente $<2$ o 2,1), 4 la misma noche de la cirugía, se comienza de nuevo con el ACO y se va controlando diariamente hasta alcanzar un INR terapéutico.

En el paciente de alto riesgo embolígeno (portadores de válvula cardíaca protésica, sobre todo mitral, 
FA coexistente, etc.), la sombra de complicaciones tromboembólicas hace que la actitud sea más conservadora. Normalmente, se tiende a reducir los niveles de ACO o a sustituir esta terapia por heparina iv., con una vida media mucha más corta y más fácilmente reversible en caso de presentarse sangrado. ${ }^{2-5,7,10,12} \mathrm{En}$ los últimos años, estamos asistiendo a un cambio en la tendencia al respecto. De una manera creciente, la mayoría de los autores abogan por una actitud más conservadora; la idea de no interferir en la terapia ACO (no suspenderla temporalmente, ni siquiera disminuir los niveles de anticoagulación) está cobrando cada vez más fuerza. ${ }^{2-7,12-20}$

Este tipo de posturas pasan, necesariamente, por optimizar las medidas de hemostasia perioperatoria, así como por llevar a cabo un adiestramiento del paciente en caso de hemorragia persistente. En este sentido, por ejemplo, Scully y Wolff recomiendan realizar la cirugía por la mañana, y así tener más tiempo durante el día para vigilar la hemostasia y, en los primeros día de la semana, para evitar problemas derivados del menor número de personal al cuidado durante el fin de semana. ${ }^{3}$ La cirugía debería llevarse a cabo usando, como anestésico local, lidocaína al 2\% con epinefrina 1:80.000 ó 1:100.000, a excepción, por supuesto, de pacientes cardiópatas, hipertensos graves o consumidores habituales de cocaína. ${ }^{2-4,7}$ Un tipo de fármacos que está ganando popularidad día a día son los antifribinolíticos, como el ácido tranexámico (Amchafibrin ${ }^{\circledR}$ ). Es un derivado sintético de la lisina que se une a los lugares de fijación de la misma en las moléculas de plasminógeno y plasmina; bloquea, así, de forma reversible está unión, inhibiendo la fibrinólisis. ${ }^{16,21}$ Administrado por vía iv., ha mostrado, en un metaanalisis, reducir de forma importante el sangrado postquirúrgico, las transfusiones requeridas e incluso la mortalidad en pacientes sometidos a cirugía cardiaca. ${ }^{19}$ En cirugía oral, de forma tópica se ha demostrado útil en, al menos, tres estudios doble-ciego controlados y randomizados. ${ }^{20,22,23}$ Además, los niveles detectados en plasma al cabo de unas horas son insignificantes (no se absorbe como colutorio oral), lo que hace que no se hayan descrito efectos sistémicos indeseables. ${ }^{21}$ Una alternativa, en países en los que no está todavía legislado el uso de este fármaco en enjuagues (EEUU, Reino Unido, etc.), es el ácido épsilon-aminocapróico (Caproamin ${ }^{\circledR}$ ) al 25\% (250 mg/ml) en enjuagues con $5-10 \mathrm{ml}^{3}$

Volviendo al manejo de la anticoagulación, Webster, en una amplia revisión bibliográfica propone una serie de directrices (si bien reconoce que faltan evidencias que apoyen las mismas). Básicamente, ante una cirugía menor (exodoncia, biopsia cutánea, etc.), con un INR $<4$ no haría falta alterar la terapia ACO. Recomienda, eso sí, extremar las medidas de hemostasia local durante y después de la intervención. Ante una cirugía mayor (parotidectomía, disección cervical, etc.). La recomendación pasa por la suspensión del ACO 3 días y el control diario del INR. Se administrarán dosis profilácticas de HBPM si INR<2,0. En caso de que, en el momento de la cirugía, el INR no haya diminuido por debajo de 1,3, se recomienda la administración de vitamina $\mathrm{K}(1 \mathrm{mg})$. La reintroducción del cumarínico se llevará a cabo desde la misma noche de la cirugía superponiendo la heparina al menos hasta 48 horas después de alcanzar un INR terapéutico. ${ }^{2}$

En otra revisión, Scully, también recomienda no modificar el ACO ante una cirugía oral limitada pero ante cirugías más amplias o pacientes con más riesgo, aboga por el ingreso hospitalario pudiendo ser necesaria la heparinización iv. Sin embargo, este autor prefiere evitar la vitamina $\mathrm{K}$ aduciendo una posible complicación a la hora de reiniciar la terapia ACO. Además, en su opinión, bastan dos días de interrupción del cumarínico antes de la cirugía. ${ }^{3}$

Tesis similares, en lo que respecta a la no interrupción del ACO en cirugías limitadas, vienen siendo sostenidas por varios autores. Así, Souto y cols., en un estudio prospectivo randomizado de pacientes anticoagulados sometidos a cirugía oral, no aprecia diferencias significativas, en cuanto a sangrado, entre el grupo en que el ACO es modificado y el que no lo es. ${ }^{24}$

Campell y Alvarado, comparan en otro estudio el sangrado postquirúrgico tras procedimientos de cirugía oral «menon» (exodoncias, alveoloplastias, etc.) entre tres grupos de pacientes $(n=35)$. El primero compuesto por pacientes anticoagulados que interrumpieron su terapia. El segundo, pacientes anticoagulados que no la interrumpieron y un tercero, control, con pacientes no anticoagulados; para valorar el sangrado se pesan las gasas y esponjas usadas en cada procedimiento y para poder compararlos las diferentes intervenciones se atribuye una puntuación por «unidades de cirugía», el estudio concluye que no se objetivan diferencias significativas y recomienda, por tanto, no modificar la ACO si INR $<3.12$

Devani, de manera similar, compara los grupos de pacientes anticoagulados, en uno de los cuales interrumpe la terapia ACO antes de la cirugía y en el otro no. No aprecia diferencias significativas. ${ }^{13}$

Beirne, llega a una conclusión parecida en su revisión: con medidas adecuadas de hemostasia local y un INR $<4$ se pueden realizar exodoncias con un umbral de seguridad suficiente evitando, así, someter al paciente a un riesgo tromboembólico innecesario. Sin embargo, también reconoce que, ante procedimientos que impliquen un mayor riesgo de sangrado, puede ser necesaria una modificación de la dosis del ACO. ${ }^{16}$

Blinder, en Israel realiza un estudio prospectivo entre 249 pacientes anticoagulados en los que se realizaron un total de 543 extracciones dentales. Todos los pacientes fueron clasificados en 5 grupos, aten- 
diendo únicamente al INR (valores entre 1,5 y >3,5). No se encuentran diferencias significativas en cuanto al sangrado entre los diferentes grupos. ${ }^{6}$

Giglio propone un protocolo sobre la decisión de modificar la terapia ACO en función del tipo de cirugía, INR y riesgo quirúrgico del paciente. Según él, procedimientos mínimamente invasivos pueden realizarse con un INR $<4$. En otros procedimientos, el INR se deberá disminuir en función del sangrado previsible y el riesgo quirúrgico del enfermo. Cualquier cirugía debe evitarse con un INR $>5.25$ Otros autores como Vongpatanasin, ${ }^{26}$ o Spandorfer, ${ }^{27}$ también señalan en este sentido que la recomendación de heparinizar no debería pesar sólo en el tipo de cirugía a realizar. Pacientes de alto riesgo embólico (válvula mitral protésica, sobre todo si coexiste FA o disfunción ventricular) deberían ser heparinizados tanto antes como después de la cirugía. Siguiendo esta línea, Mehra propone un protocolo para cambiar de ACO a heparina en pacientes de alto riesgo que van a ser sometidos a cirugía oral (Tabla 1). Lo respaldan mediante un estudio de 20 pacientes anticoagulados en los que la heparinización fue recomendada por sus cardiólogos antes de ser sometidos a cirugía oral. En el grupo en que se aplicó el protocolo se objetivaron importantes ventajas al valorar parámetros, como el número de horas requeridas para la heparinización, número de pruebas de coagulación (ambas significativamente menores en el grupo que siguió el protocolo), costo de laboratorio en dólares (de media, unos 350 más en el grupo control) o tiempo de hospitalización (de media, 2 días más en el grupo control). ${ }^{5}$

\section{Discusión}

De todo lo anterior se deduce que el manejo quirúrgico del paciente anticoagulado ha variado y está variando sustancialmente en los últimos años. Gran parte de ese cambio se debe a la introducción en 1983 del INR como método se monitorización de la terapia ACO.$^{16}$ Desde entonces, se ha ido afianzando entre los profesionales y, en la actualidad, está recomendado por la OMS como método de control para cuantificar de manera estándar los tiempos de protrombina. Esto ha permitido una unificación de criterios y ha redundado en una disminución de los niveles con que hematólogos y cardiólogos mantienen anticoagulados a sus pacientes. Pese a que, como estamos viendo en la literatura, la gran mayoría de autores utilizan este método en sus estudios, es cierto que, en sentido estricto, todavía no hay evidencias científicas de que el INR sea un método de control adecuado para realizar exodoncias y otros procedimientos; además, según una encuesta realizada a cirujanos orales y maxilofaciales norteamericanos y publicada por Troulis en el 98, el $73 \%$ de los encuestados afimaba utilizar rutinariamente el INR a la hora de intervenir a pacientes anticoagulados; sin embargo, tan sólo el 21\% usaba únicamente éste como método único. ${ }^{28}$

Bajo nuestro punto de vista el INR es un método sencillo y estandarizado que debería se usado, de forma rutinaria, por odontólogos y cirujanos maxilofaciales para controlar el nivel de anticoagulación en este tipo de pacientes. La comprensión y familiarización con este sistema está trayendo consigo, entre otras cosas, un ahorro de pruebas innecesarias.

$\mathrm{Si}$ intentamos recomendar unas normas de actuación o un protocolo uniforme a la hora de enfrentarnos a un paciente anticoagulado que vamos a intervenir, debemos tener en cuenta varios aspectos: En primer lugar hemos observado que a muchos de los artículos revisados, aunque realizados de forma brillante y con grandes dosis de sensatez, si atendemos exclusivamente a la medicina basada en la evidencia, les falta cierto rigor (bien por un número insufiente de pacientes incluidos en los estudios o bien por la dificultad que entraña el diseño de los mismos en esta materia). Así, Campbell y Alvarado en su magnífica comparación de los tres grupos, reconocen que juegan con un tamaño muestral pequeño y que el tema merece futuras investigaciones. ${ }^{12}$ Beirne, en su discusión a este estudio, aunque lo alaba en muchos aspectos, le achaca una pérdida de rigor al no cuantificar el sangrado diferido. ${ }^{29}$ De igual modo, Webster reconoce que hay limitadas evidencias para el protocolo que propone. Además, en este caso, es cierto que no hay estudios grandes randomizados que comparen diferentes regímenes preoperatorios en pacientes portadores de válvulas cardíacas protésicas; y es, precisamente, este tipo de pacientes los que tienen un mayor riesgo embolígeno. ${ }^{2}$

Aceptando la limitación de los estudios sobre esta materia, el segundo problema suscitado es la falta de consenso y la gran controversia que todo este tema continúa generando. Todo ello hace que cualquier intento de sugerir una normas de actuación deba basarse, no sólo en los estudios y revisiones publicados al respecto, sino también en el sentido común y la accesibilidad y fácil manejo de las recomendaciones. Así, a la hora de plantear una interrupción en la terapia ACO (actitud mas común hasta hace unos años), nos encontramos con dos vertientes:

- Por un lado, varios autores justifican, en cierto modo, la interrupción al considerar mínimo»el riesgo de accidente embólico. Por ejemplo, Tinker no encuentra ninguna complicación en el seguimiento de 159 pacientes con válvula protésica, en los que se modificó el ACO, sometidos a un total de 180 intervenciones ${ }^{30}$. Kearon y Hirsch justifican también una «entana» en la ACO, sin siquiera heparinizar de forma sustitutiva, en virtud del elevado riesgo de sangrado y del ínfimo riesgo tromboembólico (es cierto que hace la excepción de pacien- 
tes portadores de prótesis, con valvulopatía mitral o con disfunción ventricular). ${ }^{31}$ Eckman demuestra, incluso, un rédito económico con esta actitud. ${ }^{32}$ Butler cifra en un 0,06\% el riesgo de tromboembolismo en 5 días preoperatorios con baja anticoagulación. Aunque hubiese fenómeno de rebote, que lo pone en duda, el riesgo no sería mucho mayor de esa cifra. ${ }^{33}$ Saour en su estudio, no encuentra ninguna complicación embólica (realizando ecocardiografia y ultrosonografía Doppler), pero sí, dos episodios de sangrado postquirúrgico que motivaron ingreso hospitalario. ${ }^{17}$

- En la postura contraria, se afirma que no hay casos bien documentados de sangrados importantes en pacientes anticoagulados, dentro de limites terapéuticos y sometidos a cirugía oral pero sí, complicaciones embólicas entre los que se suspende la misma. ${ }^{34}$ Katholi, por ejemplo, reporta dos casos de embolia de desenlace fatal, 35 y Evans encuentra cuatro casos descritos en la literatura. ${ }^{4}$ Además, según Scully y Wolff, hasta el 90\% de los casos, la hemorragia se produce por causas distintas de la anticoagulación (excesivo trauma quirúrgico, infección, escasa adherencia terapéutica del enfermo a las instrucciones dadas, interacción medicamentosa, hipertensión, etc.). Según esto, un adecuado control de estos factores va a dar lugar a una importante disminución en la incidencia de sangrados postquirúrgicos. ${ }^{3}$ Una encuesta reciente realizada entre pacientes anticoagulados (algunos, muy por encima de los límites recomendados) sometidos en total a 2.400 intervenciones muestra que en tan solo 12 ocasiones $(1,3 \%)$ fueron precisas más que medidas locales para el control en la hemorragia. ${ }^{36}$ Muchos autores continúan considerando muy importante el riesgo de hipercoagulabilidad de rebote al suspender el ACO de forma brusca. ${ }^{36-}$ ${ }^{41}$ Tanto la Sociedad Europea de Cardiología como la 4a Conferencia de consenso sobre terapia antitrombótica del American College of Chest Physicians recomiendan la heparinización perioperatoria. ${ }^{2,42}$ Este se muestra también como el método preferido por un $65 \%$ de hematólogos españoles encuestados. 43

En resumidas cuentas, lo cierto es que la tendencia actual está claramente orientada a no modificar la ACO salvo en casos en que sea previsible un sangrado excesivo (bien por un exceso en la anticoagulación, por el tipo de intervención o por alteraciones concomitantes de la hemostasia). A la hora de intervenir en la ACO, nunca debe hacerse sin el concurso del hematólogo y se debe vigilar estrechamente el INR para minimizar el riesgo embolígeno.

Clasificaremos a los pacientes en función del riesgo a la hora de proponer unas directrices, esta clasificación puede hacerse únicamente atendiendo al tipo de cirugía, ${ }^{2}$ del INR, ${ }^{6} 0$, lo que en principio resulte más sensato, atendiendo a varios factores (tipo de procedimiento, INR, otro factores de riesgo, juicio clínico, etc.). ${ }^{3}$

\section{Conclusiones}

En este trabajo hemos orientado un protocolo de manejo quirúrgico en el anticoagulado que se basa, principalmente, en el INR del paciente y en el tipo de cirugía a realizar. Creemos que resume, en cierto modo, las tendencias actuales al respecto; no obstante, debe entenderse de una manera flexible ya que otros factores, como las perspectivas de vigilancia y cumplimiento terapéutico de un paciente o la coordinación con el Servicio de Hematología deberán ser tenidos en cuenta. Por tanto, en este tema, como en tantos otros, juega un papel crucial el sentido común del cirujano. Con todos estos datos podemos sugerir una actitud terapéutica:

1. Por supuesto, siempre debemos tener en cuenta que un paciente anticoagulado, independientemente de su patología de base, entraña un cierto riesgo quinúrgico.

Antes de indicar una cirugía oral, valoraremos varios puntos:

- Otras condiciones coexistentes que puedan alterar todavía más la hemostasia (enfermedad de Von Willebrand, toma de fármacos, trombocitopenia, alteraciones vasculoplaquetarias, coagulopatías, etc.)

- La prevención cobra en estos pacientes un especial protagonismo (odontología conservadora, cualquier otra alternativa al tratamiento quirúrgico).

- El INR, utilizado de manera habitual en el control periódico de una terapia ACO, se considera un buen índice a la hora de permitir al cirujano conocer, con una sola prueba, el estado de coagulación de un paciente en tratamiento con cumańnicos.

2. En el momento de la cirugía, conviene adoptar precauciones especiales:

- Se recomienda,como hemos visto, realizarla por la mañana y, a ser posible, los primeros días de la semana, sobre todo en pacientes de riesgo añadido.

- Salvo contraindicación, usaremos un anestésico local del tipo de la lidocaína al 2\% con epinefrina 1:80.000 o 1:100.000 y debemos ser especialmente cuidadosos a la hora de infiltrar para no producir un trauma añadido y sangrados incoercibles que puedan disecar tejidos y llegar a comprometer la vía aérea. En este sentido, los tejidos linguales del tercer molar inferior serán siempre más peligrosos que el manejo por vestibular. 3,44,45 
- En caso de exodoncias debemos ser parcos en la osteotomía, sobreindicando, si da lugar, la odontosección. Ante la realización de colgajos mucoperiósticos, se procurará ser lo más atraumatico posible, evitando disecar planos por los que el hematoma podrá abrirse camino hasta llegar a comprimir la vía aérea. El cureteado meticuloso de la cavidad es fundamental para evitar infecciones secundarias (causa importante de sangrado postquirúrgico). En cuanto a las suturas, son preferibles las reabsorbibles, del tipo del polyglactin 910 (Vicryl $\left.{ }^{\circledR}\right)$.

Disponemos, además, de otros medios tópicos para conseguir la hemostasia secundaría: esponjas de gelatina, trombina, colágeno (sintético o porcino), cianoacrilato, oxicelulosa (Surgicel ${ }^{\circledR}$ ). La oxicelulosa empapada en ácido tranexámico se ha mostrado especialmente útil. ${ }^{22}$ Recientemente, el sellado con fibrina (Tissucol ${ }^{\circledR}$ ) se está mostrando como una herramienta tan eficaz como la oxicelulosa, aunque con el inconve-

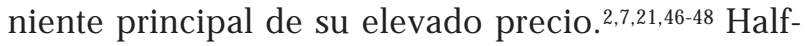
penny incluso, encuentra una menor incidencia de dolor postoperatorio que usando celulosa. ${ }^{46}$

3. De cara a la intervención o no sobre la terapia ACO, podemos subdividir a los pacientes en dos grupos, entendiendo, por supuesto, que los criterios de inclusión en uno o en otro son flexibles y dependen, en gran medida, del juicio clínico del profesional, protocolo consensuado en el hospital, etc. - Pacientes que van a ser sometidos a procedimientos de cirugía oral limitada (exodoncias 13 piezas, biopsias intraorales, etc.), con INR $<3,5$ y sin otros factores de riesgo: Se recomienda no modificar la anticoagulación, extremando, por supuesto las medidas de hemostasia local y los cuidados postquirúrgicos.

- Ante cirugías menos limitadas (poliextracciones, despegamientos palatinos, etc.), INR $>3,5$ o la existencia de otros factores de riesgo: Aquí es donde hay más controversia. Parece razonable recomendar la suspensión del ACO, dos o tres días antes de la cirugía y el ingreso hospitalario para comenzar la infusión de heparina iv (Tabla 2). Una alternativa a la heparinización en el hospital está en el uso de HBPM. De decantarse por este metodo una opción sería suspender el ACO 3 días antes del procedimiento y chequear el INR diariamente. Deben administrarse dosis profilácticas si INR $<2,0$. Algunos autores son partidarios de dar una nueva dosis profiláctica antes de la cirugía, ${ }^{7}$ pero, basándose en la predictibilidad del efecto, Todd y Roman proponen un método más sencillo: suspender el ACO y administrar HBPM 2 veces al día a dosis de $1 \mathrm{mg} / \mathrm{Kg}$ de peso. La mañana de la intervención se suspenderá la heparina volviéndose a instaurar ésta unas horas después. El ACO se reinicia al día siguiente de la
Tabla 2. Directrices para la iniciación y modificación de la terapia con heparina ${ }^{5}$

1. Interrumpir terapia ACO dos dias antes de la hospitalización.

2. Determinar APTT, INR y recuento plaquetario al ingreso.

3. Bolo de heparina iv. a dosis de $80 \mathrm{U} / \mathrm{Kg}$.

4. Comenzar la infusión iv. continua a $18 \mathrm{U} / \mathrm{Kg} / \mathrm{h}$.

5. Control APTT 6 horas después.

6. Ajustar la dosis según la siguiente escala:

APTT $<35$ s Bolo de $80 \mathrm{U} / \mathrm{Kg}$

Incremento del ritmo de infusión en $4 \mathrm{U} / \mathrm{Kg} / \mathrm{h}$.

APTT 35-45s Bolo de $40 \mathrm{U} / \mathrm{Kg}$.

Incremento del ritmo de infusión en $2 \mathrm{U} / \mathrm{Kg} / \mathrm{h}$

APTT 46-70s Sin cambios. Nivel terapéutico.

APTT 71-90s Reducir ritmo de infusión en $2 \mathrm{U} / \mathrm{Kg} / \mathrm{h}$.

APTT $>90$ s Retirar heparina 1hora. Reducir ritmo de infusión en $3 \mathrm{U} / \mathrm{Kg} / \mathrm{h}$.

intervención superponiendo ambas terapias hasta lograr un INR deseado, lo que suele demorarse en torno a tres días para la Warfarina o dos para la Cumarina. ${ }^{10}$

4. Una vez realizada la cirugía, el paciente recibirá una serie de normas. Es esencial conseguir una buena adherencia terapéutica pues de ella depende, fundamentalmente, la aparición o no de complicaciones:

- El paciente estará advertido que, ante la aparición o sospecha de hematoma (disfagia creciente, aumento de tumefacción intraoral, dificultad respiratoria, etc.) o ante un sangrado no controlable con medidas locales de presión (mordiendo una gasa durante 30 minutos), debe llamar al teléfono de emergencia que se le ha facilitado o acudir, en su defecto a un servicio de urgencias hospitalario. El cirujano, en casos de sangrado, debe limpiar bien de coágulos la zona con suero caliente. Tras identificar el punto sangrante, se puede administrar una inyección de anestésico local con epinefrina; una gasa empapada en ácido tranexámico puede aplicarse de forma compresiva sobre la herida durante 1015 min. y si persiste el sangrado, se puede dar algún punto hemostático. En caso de ausencia de control, el acetato de desmopresina (D-8DAVP) puede semos útil. Se trata de un análogo sintético de la vasopresina que induce la liberación de factores del endotelio; ofrece una alternativa a productos de transfusión a la hora de controlar sangrados rebeldes en casos de hemofilia. ${ }^{49} \mathrm{Se}$ administra en forma de spray intranasal ( 0,1 ml. que equivale a una dosis de 100-150 mcg. de fármaco).

- Se prescribirá dieta líquida y fría durante las primeras 24-48 horas.

- Los enjuagues con un antifibrinolítico durante 710 días son altamente recomendables. El mejor 
conocido es el ácido tranexámico ( Solución al 4,8-5\%, cuatro enjuagues al día de $10 \mathrm{ml}$. durante 2 minutos). Una alternativa es el ácido épsilon-aminocapróico al 25\% (enjuagues de 5-10 $\mathrm{ml})$.

- En cuanto a la analgesia utilizaremos paracetamol y/o un opiáceo suave del tipo de la codeína. En la medida de lo posible no se debe prescribir AAS u otros AINES por el evidente riesgo aumentado de sangrado al interferir con la agregación plaquetaria.

- Otro importante debate se abre en cuanto a la prescripción de antibióticos: Por un lado es cierto que se han publicado varios casos de sangrado inducido por antibióticos tras procedimientos dentales en pacientes anticoagulados. ${ }^{15,50,51}$ Ciertos antibióticos entre los que se incluyen quinolonas, ciertas sulfamidas, eritromicina, amoxicilina-clavulánico, cefalosporina, ampicilina, etc, interfieren con los derivados cumarínicos. Sin embargo, otros estudios no han encontrado relación entre la toma de antibiótico y el incremento de riesgo de sangrado. ${ }^{4}$ Algunos autores recomiendan una terapia con penicilina $\mathrm{V}$ (250-500 $\mathrm{mg} / 6 \mathrm{~h}$ ) o clindamicina (300 mg/ 8h) durante una semana en aras de prevenir la infección, importante factor inductor de la fibrinólisis. ${ }^{3}$

5. Todo lo que hemos expuesto hasta ahora hace referencia a la cirugía programada; en una situación de emergencia, el planteamiento, por supuesto, varía. - Normalmente, ante un planteamiento quirúrgico y, si el estado del paciente nos permite diferir el abordaje unas horas, podemos revertir parcialmente el estado de anticoagulación mediante una dosis baja ( $1 \mathrm{mg}$ ) de vitamina $\mathrm{K}$ iv. Después, durante la cirugía, continuaremos la administración de vitamina $\mathrm{K}$ para mantener la reversión.

- Si es necesaria una intervención inmediata, intentaremos corregir la anticoagulación con plasma fresco congelado o concentrados de complejo de protrombina (si está disponible en el hospital). De todas formas, en este tipo de situaciones, siempre se debería consultar a un hematólogo. ${ }^{2}$

6. Aunque no es, en sentido estricto, objeto de nuestro estudio, no queremos finalizar la exposición sin hacer una breve reseña sobre la actitud quirúrgica ante un paciente antiagregado. El ácido acetil-salicílico (AAS) y otros AINES tienen un importante efecto antiagregante, fundamentalmente al interferir con la ciclooxigenasa plaquetaria.1,52,53 Un paciente en tratamiento con este tipo de fármacos tendrá un TH prolongado sin verse alterados (en principio) los tiempos de coagulación. En el caso del AAS, este efecto es irreversible, la acetilación se mantendrá hasta que la plaqueta sea recambiada, por tanto, el estado de antiagregación durará en torno a una semana (que corresponde a la vida media plaquetaria). ${ }^{1}$ En cuanto a otros efectos, cabe citar que el diclofenaco ha demostrado retrasar la neoformación ósea postexodoncia en animales de experimentación, lo que puede tener importantes implicaciones fisiopatológicas en humanos. ${ }^{54}$

Ante un paciente antiagregado $\mathrm{y}$, siguiendo a Scully, ${ }^{3}$ se puede recomendar la siguiente actitud:

- Si recibe una dosis menor de $100 \mathrm{mg}$ diarios, pueden realizarse exodoncias no complicadas de 13 piezas. Por supuesto, extremaremos las medidas de hemostasia secundaria y se recomienda el uso de ácido tranexámico en enjuagues después de la cirugía.

- Ante cirugías de mayor envergadura, o dosis supe riores de AAS, debemos determinar el TH; si supera los $20 \mathrm{~min}$, postpondremos el acto quirúrgico una semana. En caso de que sea una emergencia, podemos utilizar D-8-DAVP, siempre bajo supervisión del hematólogo.

\section{Bibliografía}

1. Arias-Díaz J, Giner Díaz J. Hemorragia y hemostasia quirúrgica. En: Arias J (ed). Propedéutica quirúrgica. Ed. Tébar, Madrid 2003 (En prensa).

2. Webster K, Wilde J. Management of anticoagulation in patients with prosthetic heart valves undergoing oral and maxillofacial operations. Br J Oral Maxillofac Surg 2000; 38:124-6.

3. Scully C, Wolff A. Oral surgery in patients on anticoagulant therapy. Oral Surg Oral Med Oral Pathol Oral Radiol Endod 2002;94:57-641.

4. Evans IL, Sayers MS, Gibbons AJ, Price G, Snooks H, Sugar AW. Can warfarin be continued during dental extraccion? Results of a randomized controlled trial. J Oral Maxillofac Surg 2002;40:248-52.

5. Mehra P, Cottrell DA, Bestgen SC, Booth DF. Management of heparin therapy in the high-risk, chronically anticoagulated, oral surgery patient: a review and a proposed nomogram. J Oral Maxillofac Surg 2000;59:198-202.

6. Blinder D, Manor Y, Martinowitz U, taicher S. Dental extractions in patients maintained on oral anticoagulant therapy: comparison of INR value with occurrence of postoperative bleeding. Int J Oral Maxillofac Surg 2001;30:518-21.

7. Schardt-Sacco D. Update on coagulopathies. Oral Surg Oral Med Oral Pathol Oral Radiol Endod 2000;90:559-63.

8. Mismetti P, Laporte-Simitsidis S, Tardy B y cols. Prevention of venous thromboembolism in internal medicine with unfractionated of low molecular weight heparins: a meta-analysis of randomized clinical trials. Thromb Haemost 2000;83:14-9.

9. Kakkar V, Howes J, Sharma V, Kadziola Z. A comparative, double blind, randomized trial of a new second generation LMWH and UFH in the prevention of pos- 
toperative venous thromboembolism. Thromb Venous 2000;83:523-9.

10. Todd D, Roman A. Outpatient use of low-molecular weight heparin in an anticoagulated patient requiring oral surgery: case report. J Oral Maxillofac Surg 2001;59: 1090-2.

11. Majerus P, Dalcette B, Hermans M, Pourtois M, Capel P. Variations in fibrinolytic activity of human whole saliva. Eur J Oral Sci 1996;104:341-5.

12. Campbell J, Alvarado F, Murray R. Anticoagulation and minor oral surgery: should the anticoagulation regime be altered?. J Oral Maxillofac Surg 2000;58:131-5.

13. Devani P, Lavery KM, Howell CJT. Dental extractions in patients on warfarin: is alteration of anticoagulant regime necessary?. Br J Oral Maxillofac Surg 1998;36:10711.

14. Zusman SP, Lusting JP, Bin Nun G. Cost evaluation of two methods of post tooth extraction hemostasis in patients on anticoagulant therapy. Community Dental Health 1993;10:167.

15. Aldous JA, Olson CJ. Managing patients on warfarin therapy: a case report. Spec Care Dentist 2001;21:109-12.

16. Beime OR, Koehler JR. Surgical management of patients on warfarin sodium. J Oral Maxillofac Surg 1996;54:1115-8.

17. Saour JM, Ali HA, Mammo LA y cols. Dental procedure in patients receiving oral anticoagulation therapy. J Heart Valve Dis.1994;3:315.

18. Bemardoni-Socorro C, Arteaga Vizcaino M, y cols. Mouth eashing with tranexamic acid in patients treated with oral anticoagulants subjected to oral surgery procedures. Invest clin 1998;39:77-83.

19. Dunn CJ, Goa KL. Tranexamic acid: a review of its use in surgery and other indications. Drugs 1999;57:1005-32.

20. Borea G, Montebugnoli L, Capuzzi P, Magelli C. Tranexamic acid as a mouthwash in anticoagulant-treated patients undergoing oral surgery. An alternative method to discontinuing anticoagulant therapy. Oral Surg Oral Med Oral Pathol 1993;75:29-31.

21. GasparR, Brenner B, ArdekianL, Pele M, Laufer D. Use of tranexamic acid mouthwash to prevent postoperative bleeding in oral surgery patients on oral anticoagulant medication. Quintessence Int 1997;28:375-9.

22. Ramstrom G, Sindet-Pedersen S, Hall G, Blomback M, Alexander U. Prevention of postsurgical bleeding in oral surgery using tranexamic acid without dose modification of oral anticoagulants. J Oral Maxillofac Surg 1993;51: 1211-6.

23. Sindet-Pedersen S. Haemostasis in oral surgery: the possible pathogenetic implications of oral fibrinólisis on bleeding. Experimental and clinical studies of the haemostatic balance in the oral cavity, with particular reference to patients with aquired and congenital defects of the coagulation system. Dan Med Bull 1991;38:427-43.

24. Souto JC, Oliver A, Zuazu-JausoroI y cols. Oral surgery in anticoagulated patients without reducing the dose of oral anticoagulant: a prospective randomised study. J Oral Maxillofac Surg 1996;54:27-32.
25. Giglio J. Complications in dentoalveolar surgery. In: Kwon P, Laskin D (eds.). Clinicians manual of oral and maxillofacial surgery (III). Q uintessence Publishing 1997;p: 271-2.

26. Vongpatanasin W, Hillis LD, Lange RA. Prosthetic heart valves. N Engl J Med 1996;335:407-16.

27. Spandorfer J, Merli G, Lowson SM y cols. Anticoagulation and elective surgery(2). N Engl J Med 1997;337:938-40.

28. Troulis MJ, Head TW, Lecrerc JR. Dental extractions in patients on an oral anticoagulant: a survey of practices in North America. J Oral Maxillofac Surg 1998;56:9147.

29. Beirne R. Anticoagulation and minor oral surgery: should the anticoagulation regimen be altered?. Discusión. J Oral Maxillofac Surg 2000;58:135-6.

30. Tinker JH, Tarhan S. Discontinuing anticoagulant therapy in surgical patients with cardiac valve protheses. J Am Med Assoc 1978;239:738-9.

31. Kearon C, Hirsch J. Management of anticoagulation before and after elective surgery. NEngl J Med 1997;336:150611.

32. Eckman MH, Beshansky JR, Durand-Zaleski I, Levine HJ, Pauker SG. Anticoagulation for noncardiac procedures in patients with prosthetic heart valves: does low risk mean high cost?. J Am Med Assoe 1990;263:1513-21.

33. Buttler AC, Tait RC. Management of oral anticoagulantinduced intracranial haemorrhage. Blood Rev 1998;12: 35-44.

34. Wahl MJ. Dental surgery in anticoagulated patients. Arch Intern Med 1998;158:1610-6.

35. Katholi RE, Nolan SP, McGuire LB. Living with prosthetic hesrt valves: subsequent non-cardiac operations and the risk of thromboembolism or haemorrage. Am Heart J 1976;92:162-7.

36. Wahl MJ. Myths of dental surgery in patients receiving anti-coagulant therapy. J Am Dent Assoc 2000;131:77-81.

37. Poller L, Tomson J. Evidence for «rebound»hypercoagulability after stopping anticoagulants. Lancet 1964;ii: $62-4$.

38. Grip L, Blomback M, Schulman S. Hypercoagulable state and thromboembolism following warfarin withdrawal in post myocardial infarct patients. Eur Heart J 1991;12:1225-33.

39. Haremberg J, Haas R, Zimmerman R. Plasma hypercoagulability after termination of oral anticoagulants. Thromb Res 1983;29:627-33.

40. Mulligan R. Response to anticoagulant drug withdrawal. J Am Dent Assoc 1987;115:435-8.

41. Marshall J. Rebound Phenomena after anticoagulant therapy in cerebrovascular disease. Circulation 1963;28:32932.

42. Ad Hoc committee of the Working Group on Valvular Heart Disease. European Society of Cardiology. Guidelines for the prevention of thromboembolic events in valvular heart disease. J Heart Valve Dis 1993;2:398-410.

43. Diz Dios P, Fernández Feijoo J. Tooth removal and anticoagulant therapy. (Carta). Oral Surg Oral Med Oral Pathol Oral Radiol Endod 2001;92:248. 
44. Scully C, Cawson RA. Medical problems in dentistry. $4^{\text {th }}$ ed. Oxford, London, and Boston: Wright; ButterworthHeinemann, 1997.

45. Berini Aytés L, Gay Escoda C. Técnicas anestésicas en cirugía bucal. En: Gay Escoda C, Berini Aytés L. Cirugía Bucal. Madrid. Ed. Ergón S.A. 1999.

46. Halfpenny W, Fraser Js, Adlam DM. Comparison of 2 hemostatic agents for the prevention of postextraction haemorrhage in patients on anticoagulants. Oral Surg Oral Med Oral Pathol Oral Radiol Endod 2001;92:257-9.

47. Gibbons AJ, Evans IL et al. Warfain and extractions. British Dent J 2002;193.

48. Bodner L, Weinstein JM, Baumgarten AK. Efficacy of fibrin sealant in patients on various level of oral anticoagulant undergoing oral surgery. Oral Surg Oral Med Oral Pathol Oral Radiol Endod 1998;86:421-4.
49. DDAVP in haemophilia and Von Willebrand's disease. Lancet 1983;2:774-5.

50. Wood GD, Deeble T. Warfarin: dangers with antibiotics. Dental Update 1993;20:350-3.

51. Bandrowsky T, Vorono AA, Borris TJ, Marcantoni HW. Amoxicilin-related postextraction bleeding in an anticoagulated patient with tranexamic acid rinses. Oral Surg Oral Med Oral Pathol Oral Radiol Endod 1996;82:610-2.

52. Parfitt K (ed). Martindale. The complete drug reference, 32nd ed. Ed Pharmaceutical Press. London 1999.

53. Ardekian L, Gaspar R, Peled M, Brener B, Laufer D. Does low-dose aspirin therapy complicate oral surgical procedures?. J Am Dent Assoc 2000;131:331-5.

54. Yugoshi LI, Sala MA, Brentegani LG, Lamano Carvalho TL. Histometric study of socket healing after tooth extraction in rats treated with diclofenac. Braz DentJ 2002; 13:92-6. 